\title{
Constrained Utility Maximization in Dual-Functional Radar-Communication Multi-UAV Networks
}

\author{
Xinyi Wang, Graduate Student Member, IEEE, Zesong Fei, Senior Member, IEEE, \\ J. Andrew Zhang, Senior Member, IEEE, Jingxuan Huang, and Jinhong Yuan, Fellow, IEEE
}

\begin{abstract}
In this paper, we investigate the network utility maximization problem in a dual-functional radar-communication multi-unmanned aerial vehicle (multi-UAV) network where multiple UAVs serve a group of communication users and cooperatively sense the target simultaneously. To balance the communication and sensing performance, we formulate a joint UAV location, user association, and UAV transmission power control problem to maximize the total network utility under the constraint of localization accuracy. We then propose a computationally practical method to solve this NP-hard problem by decomposing it into three sub-problems, i.e., UAV location optimization, user association and transmission power control. Three mechanisms are then introduced to solve the three sub-problems based on spectral clustering, coalition game, and successive convex approximation, respectively. The spectral clustering result provides an initial solution for user association. Based on the three mechanisms, an overall algorithm is proposed to iteratively solve the whole problem. We demonstrate that the proposed algorithm improves the minimum user data rate significantly, as well as the fairness of the network. Moreover, the proposed algorithm increases the network utility with a lower power consumption and similar localization accuracy, compared to conventional techniques.
\end{abstract}

Keywords: multi-UAV network, dual-functional radarcommunication, user association, power control, joint communication and radar sensing

\section{INTRODUCTION}

Unmanned aerial vehicle (UAV) is regarded as an important component for future mobile networks that can provide both ubiquitous communication [1] and radar sensing functions, thanks to its flexible on-demand deployment [2] and freedom in trajectory design [3]. Particularly in the emergency situations, such as disaster relief or battlefield, UAVs can not only provide instant communications for users [4], but also localize targets or sense the environment to avoid obstacle and potential attack [5].

This work was supported in part by National Natural Science Foundation of China under Grant U20B2039 and inpart by National Natural Science Foundation of China under 61871032.

Xinyi Wang, Zesong Fei and Jingxuan Huang are with the School of Information and Electronics, Beijing Institute of Technology, Beijing 10081, China. E-mail:bit_wangxy@163.com, feizesong@bit.edu.cn, 1120121556@bit.edu.cn.

J. Andrew Zhang is with the School of Electrical and Data Engineering, University of Technology Sydney, NSW, Australia 2007. Email:Andrew.Zhang@uts.edu.au

Jinhong Yuan is with the School of Electrical Engineering and Telecommunications, University of New South Wales, Sydney, NSW 2052, Australia E-mail:j.yuan@unsw.edu.au
Although the advantages of a single UAV have been demonstrated in enhancing the performance of wireless networks [8]-[10], it has limited capability in general and may not satisfy the communication requirement due to the size, weight, and power (SWAP) constraints [11]. Besides, in single-site positioning, multiple distance measurements from a single UAV on same targets may lead to large accumulated errors [6]. A multi-UAV system can potentially overcome these problems. Recently, a promising technique is proposed to enable highperformance communication and sensing via the multi-UAV network [7]. There have been many works aiming to enhance the performance of multi-UAV networks from the perspective of communications [11]-[13] or sensing [15]-[18], separately. For communications, the user scheduling and association, UAV trajectory, and transmission power were jointly optimized in a multi-UAV enabled downlink wireless communication system in [11]. To efficiently solve the mixed integer nonconvex optimization problem, an iterative algorithm was proposed. In [12], a joint user association and power control framework was presented for downlink transmission in UAVenabled cellular networks, where the matching theory and successive convex approximation (SCA) were utilized to solve the user association subproblem and power control subproblem iteratively. In [13], the authors proposed a utility-aware transmission protocol for a UAV-assisted communication system to maximize the UAV utility, where a single UAV serves multiple heterogeneous users. For radar sensing, based on chaotic ultra wide band-multiple input multiple output (UWBMIMO) waveform, a cognitive detect-and-avoid radar system was designed to assist autonomous UAV navigation in [15]. In [16], a distributed UAV path optimization algorithm based on the gradient descent method was developed to improve angle of arrival (AOA) target localization performance. In [17], a cooperative network platform and system architecture of multiUAV surveillance was proposed, based on which the resource scheduling and task assignment scheme was designed. Furthermore, in [18], the authors put forth the idea of dynamic radar network composed of UAVs for tracking malicious UAVs with high accuracy. However, due to the SWAP constraints for UAVs, it is challenging to install both communication system and radar system. Meanwhile, deploying a large number of UAVs, in which some provide communication services while the others perform radar sensing, will not only introduce co-channel interference between communication systems and radar systems, but also increase the resource consumption. 
Joint communication and radar sensing (JCAS) [26]-[28], also known as dual-functional radar-communication (DFRC) [22]-[25], can be a potential solution to these problems. In DFRC, a single transmitted signal is used, and a majority of hardware and signal processing are shared between communication and radar. Thus, the payload and resource usage can be minimized. In [22], the authors developed a dual-function system with joint radar and communication platforms, where sidelobe control of the transmit beamforming was utilized to enable communication links. In [23], the authors proposed to use a single transmitter with multiple antennas to communicate with downlink cellular users and detect radar targets simultaneously. In [24], the authors studied the performance trade-off between radar and communication, and proposed a DFRC MIMO system to minimize the downlink multiuser interference under both a constant modulus constraint and a similarity constraint with respect to referenced radar signals. In [25], the authors developed a beampattern to enhance the radar sensing performance while guaranteeing the performance of the downlink communications for the DFRC system. In [26], an OFDM system for simultaneous radar and communication operations was designed, and the characteristics of OFDM signals were utilized in radar processing to overcome the typical drawbacks of correlation based processing. In [27], a novel multibeam framework that allows seamless integration of communication and sensing was proposed. In [28], the authors developed closed-form solutions for optimizing the coefficients in the analog antenna arrays to generate a multibeam for joint communication and radio sensing. Besides, the authors in [40] proposed a new technique for embedding communication information into MIMO radar waveform via sparse antenna array. In [41], the authors investigated the power minimization issue in DFRC system via joint subcarrier assignment and power allocation. Then in [42], the authors proposed an efficient algorithm to minimize the transmitting power under the constraints of target detection performance and information rate. Furthermore, in [43], the wireless powered DFRC system was studied. In the system, an energy minimization problem was formulated, and the optimal solution was obtained by using semidefinite relaxation and auxiliary variable methods.

By leveraging the advantages of multi-UAV network and DFRC systems, the performance of communications and radar sensing can be improved with reduced resource consumption. However, a number of important issues need to be addressed, such as the UAV locations, transmission power allocated to each UAV. In this paper, we study a general multi-UAV enabled DFRC system, where multiple UAVs are employed to simultaneously serve a group of communication users in a given area and cooperatively sense the target area. In particular, we investigate the deployment and resource allocation schemes for the multi-UAV network to improve the communication performance under required radar sensing performance.

We assume that the UAVs act as aerial relays and provide communication links for the ground users, using the predesigned DFRC waveform. In the meantime, they receive the echos of the DFRC waveform and perform radar detection to sense the target area. Since the UAVs work in a cooperative way, they can be viewed as a distributed MIMO radar such that the object in the target area can be localized. Without loss of generality, we consider that all UAVs share the same frequency band.

Focusing on the downlink transmission from the UAVs to ground users with required localization accuracy for radar sensing, we aim at maximizing the network utility under the Cramer-Rao bound (CRB) constraint of localization by jointly optimizing the UAV locations, user association, and the transmission power. The network utility metric takes both fairness and total data rate into consideration, and is more appropriate than the pure data rate metric in ad-hoc type of networks such as the multi-UAV network here [31]. Such a joint optimization problem is practically appealing, but has not been investigated in the literature to our best knowledge. On one hand, by properly designing the locations of UAVs and user association, the co-channel interference can be effectively alleviated, thus increasing the network utility. On the other hand, by optimizing the transmission power based on the designed UAV locations, not only the co-channel interference can be decreased, the localization accuracy requirement for radar sensing can also be satisfied. However, such a joint UAV location, user association and transmission power optimization problem is non-trivial to solve. This is because the UAV location, user association, and transmission power are coupled with each other in our considered problem. In this paper, we propose to firstly utilize a spectral clustering method to cluster users into different groups, and design the UAV locations accordingly. Then, user association and the transmission power are optimized by fixing each other iteratively. For user association with fixed transmission power, a coalition formation game is utilized, while for transmission power with fixed user association, we apply the successive convex approximation (SCA) technique to solve it approximately.

The main contributions of this paper can be summarized as follows.

(1) We introduce a DFRC multi-UAV network (DFRC-MUN) where multiple UAVs simultaneously serve a group of communication users and cooperatively sense the targets. We propose a multi-UAV cooperatively communication and radar sensing protocol, and formulate a joint UAV location, user association, and UAV transmission power optimization problem to maximize the network utility under the CRB constraint of localization accuracy.

(2) We propose a sub-optimal method to solve the formulated NP-hard problem. Firstly, we apply the spectral clustering method to cluster users and determine the UAV locations accordingly. The clustering result provides an initial user association strategy. Then we decompose the joint user association and UAV transmission power optimization problem into two sub-problems and solve them alternately. For the user association sub-problem, we utilize the coalition formation game to determine the set of users served by each UAV. For the UAV transmission power optimization sub-problem, the SCA technique is introduced as a solution.

(3) We compare the network utility as well as minimum user data rate performance of the proposed algorithm 


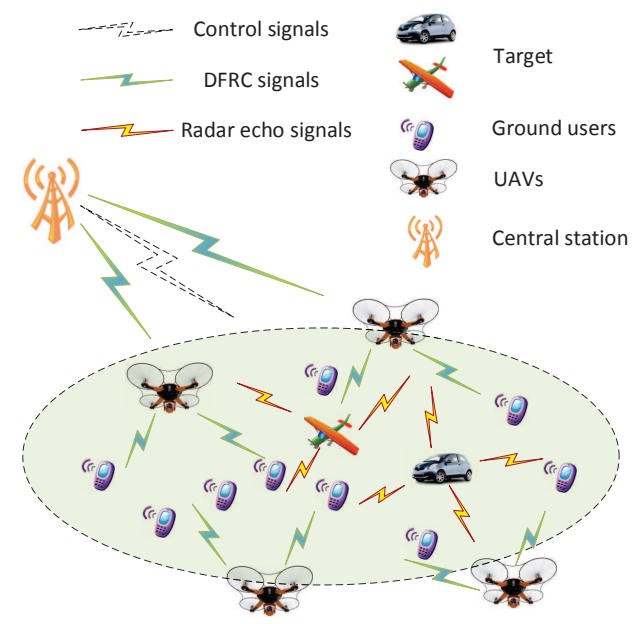

Fig. 1. System model of DFRC-MUN

with conventional schemes and a state-of-the-art scheme through simulation. Results validate the effectiveness of the proposed algorithm, and show that the proposed algorithm is able to converge within only a few iterations and significantly improve the minimum user rate and network utility with lower power consumption and similar localization accuracy.

The rest of this paper is organized as follows. In Section II, we present the system model of DFRC-MUN and the preliminaries. In Section III, we formulate a problem to maximize the total network utility by jointly optimizing UAV locations, user association, and UAV transmission power. The proposed optimization algorithms are presented in Section IV, followed by the overall algorithm and convergence analysis. Simulation results are presented in Section V. In Section VI, we conclude the paper.

Notations: Unless otherwise specified, matrices are denoted by bold uppercase letters (i.e., $\boldsymbol{\Lambda}$ ), bold lowercase letters are used for vectors (i.e., $\boldsymbol{p}$ ), scalars are denoted by normal font (i.e., $\left.\lambda_{i, k}\right), \log (\cdot)$ represents the logarithm based on $e .(\cdot)^{T}$ stands for transpose.

\section{SySTEM MOdEL}

In this section, we first describe the DFRC-MUN, where multiple UAVs simultaneously provide downlink communications for ground users and act as distributed MIMO radars. Subsequently, we introduce the preliminaries for both communication channel model and MIMO radar.

\section{A. System Description}

We consider a DFRC-MUN as shown in Fig. 1, which consists of one central station (CS), $K$ UAVs, with the set denoted by $\mathcal{K}=\{1,2, \cdots, K\}$, and $N$ ground users, with the set denoted by $\mathcal{N}=\{1,2, \cdots, N\}$. The CS broadcasts the communication information to all UAVs via DFRC signals. The UAVs then relay the signals from CS to provide downlink communication to ground users. Meanwhile, the UAVs cooperatively perform radar sensing using the downlink DFRC signals. Following the assumption in [11], all UAVs

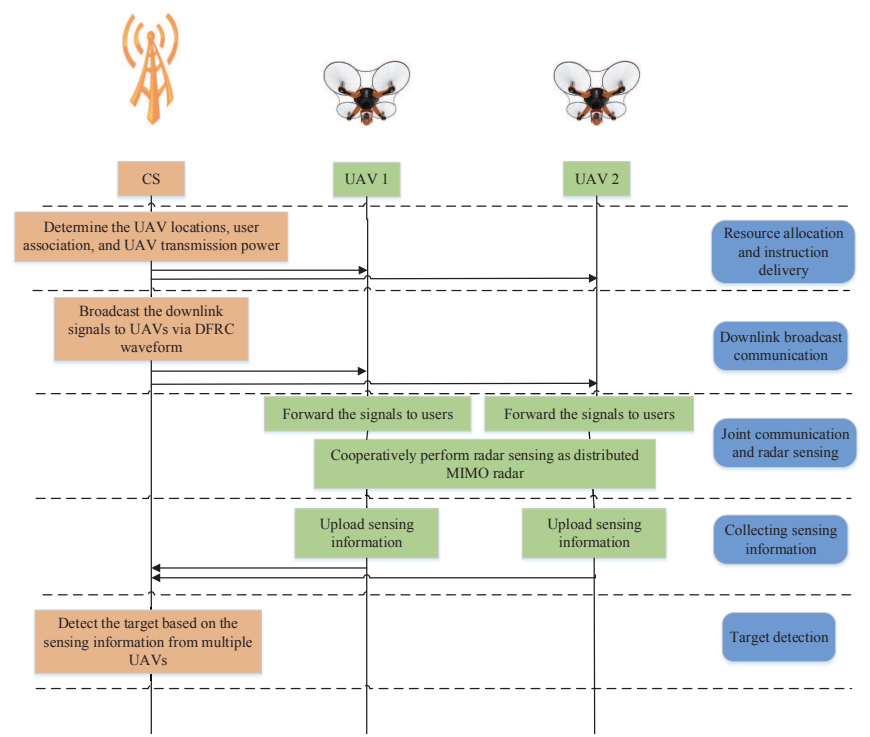

Fig. 2. Multi-UAV cooperatively communication and radar sensing protocol

are assumed to fly at a fixed altitude $H$ above ground, and the horizontal coordinate of each ground user $n$ is fixed at $\left[u_{n}, v_{n}\right]$. To fulfill the varied requirements of users, we assume that the UAV locations, user association, and UAV transmission power are adjusted periodically, at an interval in the order of sub-second, according to the instructions of the CS. Note that the locations of both UAVs and ground users are assumed to remain almost unchanged during the short period; thus, the channel remain nearly unchanged, making it possible to obtain channel state information via channel estimation based on the pilot signals received before. Furthermore, thanks to the development of power management integrated circuits [47], the UAV transmission power can also be optimized based on the channel state information.

The detailed multi-UAV communication and radar sensing protocol that simultaneously supports communication and sensing is illustrated in Fig. 2. In each time slot, the CS first determines the resource allocation strategy and delivers the instructions to each UAV. It then sends downlink signals to UAVs via DFRC waveform. Afterwards, the UAVs relay the signals to users according to the instructions. Each UAV communicates with its serving users via frequency division multiple access (FDMA). Meanwhile, the UAVs perform radar sensing using their transmitted signals, i.e., each UAV receives the echo signals that are transmitted by itself as well as any other UAV and directly reflected by targets. Since the instructions and signals are both broadcasted to all UAVs, we assume that the signals transmitted by each UAV are known to the other UAVs and stored as the template signals. The received signals are then combined with the template signals by a mixer to obtain sensing information. More specifically, the distance and radial velocity information can be retrieved from the propagation delay and phase differences among different received signal sequences, respectively. Finally, all UAVs upload the sensing information to the CS, and the CS determines the relative locations of targets with respect to UAVs. In this sense, the spatial diversity of the targets' 
radar cross section (RCS) can be captured by UAVs, and the spatially distributed antennas among all UAVs can be viewed as a distributed multiple-input multiple-output (MIMO) radar [18]. An explicit method to jointly estimate the target position and velocity for distributed MIMO radar system was proposed in [50], which is also applicable to our considered DFRCMUN. Note that the locations of UAVs can be obtained via Global Navigation Satellite System; thus, the locations of targets can be determined. For more details of multi-UAV radar sensing network, we refer readers to [18].

Remark 1. In this paper, we mainly consider the radar sensing based localization. There also exist some other techniques for localization, such as received signal strength (RSS) based localization. The advantage of radar based localization over other techniques is its insensitivity to multi-path and weather impacts, thanks to the pulse compression techniques [18]. Therefore, the DFRC-MUN considered in this paper is applicable to localizing both aircrafts and ground vehicles. For the former case, there exist no obstacles; while for the latter case, the propagation path of radar signals is also dominated by Line-of-Sight (LoS) links.

Remark 2. As a DFRC system, it is required that all UAVs are able to simultaneously transmit DFRC signals and receive echo signals. In other words, the full-duplex transmission/reception capability is required. Although the signals transmitted by UAVs are data-bearing, the data are not required for radar signal processing. Therefore, the fullduplex implementation required in this paper is the same as that for conventional radar system. There have been many works for self-interference mitigation in the field of radar signal processing. Moreover, practical measurements have been done in [49], which validated that the performance of OFDM radar can be efficiently improved by combining passive isolation [45] and active self-interference cancellation [46]. The techniques are also applicable to our considered DFRC$M U N$.

The role of the CS is two-fold. On one hand, to mitigate the co-channel interference to ground users as well as guaranteeing location accuracy, the CS optimizes the resource allocation strategy. On the other hand, the CS collects the sensing information from all UAVs and performs radar signal processing to achieve high resolution.

To improve the communication performance in terms of network utility as well as guaranteeing location accuracy, in this paper, we study the optimization of UAV locations, UAV transmission power, and user association, i.e., determining the sets of ground users that are served by different UAVs. To specify the user association, we define a $K \times N$ indicator matrix $\Lambda$, where the element at the $k$ th row and $n$th column is denoted as $\lambda_{k, n}$. The assignment index $\lambda_{k, n}$ indicates whether user $n$ is served by UAV $k$ or not:

$$
\lambda_{k, n}= \begin{cases}1, & \text { if user } n \text { is served by } \operatorname{UAV} k ; \\ 0, & \text { otherwise. }\end{cases}
$$

We assume that each UAV is able to serve multiple users, while each user is only served by one UAV, which yields the following constraints

$$
\begin{array}{r}
\lambda_{k, n} \in\{0,1\}, \quad \forall n \in \mathcal{N}, \quad \forall k \in \mathcal{K}, \\
\sum_{k=1}^{K} \lambda_{k, n}=1, \quad \forall n \in \mathcal{N}
\end{array}
$$

The transmission power set of all UAVs is denoted by $\boldsymbol{p}=$ $\left[p_{1}, \cdots, p_{K}\right]^{T}$, where the $k$ th element represents the power of UAV $k$. And the horizontal coordinates of UAVs are denoted by $[\boldsymbol{x}, \boldsymbol{y}]=\left[x_{1}, \cdots, x_{K} ; y_{1}, \cdots, y_{K}\right]^{T}$, where the $k$ th row, $\left[x_{k}, y_{k}\right]$, denotes the coordinate of UAV $k$.

\section{B. Preliminaries}

Before determining the user association and transmission power, the CS needs to obtain the channel state information (CSI) between UAVs and users to improve the communication performance. Note that the system considered in this paper is a quasi-static network, such that the communication channels between UAVs and users are quasi-static; thus, the acquisition of CSI can be achieved via channel estimation techniques based on the pilot signals received before. For the case that the CSI is imperfect, robust power control [37] can be utilized to guarantee the performance. Moreover, thanks to the high altitude of UAVs, the communication channels between UAVs and ground users are largely dominated by LoS links, and the impact of multi-path channel on CSI as well as communication performance decreases with the increase of UAV altitude. Therefore, for simplicity, we assume that the communication links from the UAV to the ground users are dominated by the LoS links where the channel quality depends mainly on the UAV-user distance [11]. Also note that in radar sensing, the localization is based on the round trip delay estimation of echo signals directly reflected from the target, and the impacts of multi-path propagation can be mitigated via pulse compression technique. Therefore, free-space path loss model is applicable to both communications and radar sensing. The channel gain from UAV $k$ to user $n$ follows the free-space path loss model, which can be expressed as

$$
h_{k, n}=\sqrt{\rho_{0} d_{k, n}^{-2}},
$$

where $\rho_{0}$ denotes the channel power at the reference distance $d_{0}=1 \mathrm{~m}$, and $d_{k, n}$ is the distance from UAV $k$ to user $n$, which can be expressed as

$$
d_{k, n}=\sqrt{H^{2}+\left(x_{k}-u_{n}\right)^{2}+\left(y_{k}-v_{n}\right)^{2}} .
$$

Note that the network utility model was applied to define the fairness in multiple resource-allocation problems in [31]. In this paper, a proportionally fair network utility optimization framework of maximizing the sum log-utility across all the users [19] is adopted. Assuming constant channels and flat transmission power spectral density, for a given set of users associated with one UAV, the round-robin scheme among these users is the proportionally fair schedule [20]. Therefore, we assume that each user served by the same UAV is assigned equal bandwidth. The load of UAV $k$ is represented by the number of users associated with UAV $k$, which is denoted by 
$\beta_{k}=\sum_{n \in \mathcal{N}} \lambda_{k, n}$. The effective rate of user $n$ associated with $\mathrm{UAV} k$ is obtained by

$$
R_{k, n}=\frac{B}{\beta_{k}} \log _{2}\left(1+\Gamma_{k, n}\right),
$$

where $B$ represents the total bandwidth, and $\Gamma_{k, n}$ represents the signal-to-interference-and-noise ratio (SINR) of the signal received by user $n$ from UAV $k$ under the free-space path loss channel model and is given as follows

$$
\Gamma_{k, n}=\frac{p_{k} h_{k, n}^{2}}{\sum_{k^{\prime} \in \mathcal{K} \backslash k} p_{k^{\prime}} h_{k^{\prime}, n}^{2}+N_{0}},
$$

where $N_{0}$ denotes the noise power spectral density, and $p_{k}$ denotes the transmission power of UAV $k$. The utility is calculated as $U\left(R_{n}\right)=\log \left(R_{\mathcal{K}_{n}, n}\right)$ [19], where $\mathcal{K}_{n}$ denotes the index of the UAV serving user $n$, and $\left(R_{\mathcal{K}_{n}, n}\right)$ denotes the data rate of user $n$.

As for radar sensing, a target with a center of mass located at position $\left(x_{t}, y_{t}\right)$ is assumed [21], and the UAVs attempt to localize and track the target cooperatively. The variation in the location of the target center and the size of target, as viewed by the UAVs, are assumed to be small with respect to the UAV system resolution capabilities. The UAV system is tracking the target's location and has coarse estimates for unknown parameters, such as the target radar cross-section, from previous cycles. The search cell is confined to $\left(x_{t} \pm\right.$ $\left.\delta c / B, y_{t} \pm \delta c / B\right)$, where $\delta$ is an integer, $c$ is the speed of light, and $B$ is the signal bandwidth, as defined in [29].

The baseband representation of the signal received at UAV $n$ and transmitted from UAV $m$ is

$$
r_{m, n}(t)=\sqrt{p_{m}} \alpha_{m, n} l_{m, n} s_{m}\left(t-\tau_{m, n}\right)+w(t),
$$

where $\alpha_{m, n}$ is the target RCS, $l_{m, n}$ represents the signal strength variation due to path loss effects, $\tau_{m, n}$ denotes the propagation time of the signal transmitted by UAV $m$, reflected by the target, and received by $\operatorname{UAV} n$, and $w(t) \sim \mathcal{C N}\left(0, \sigma_{w}^{2}\right)$ represents circularly symmetric complex Gaussian white noise.

For the unbiased (or asymptotically unbiased) parameter estimator in radar sensing, the CRB could provide a lower bound for the mean square error (MSE). The MSE of the maximum likelihood estimator approaches the CRB when the SNR is sufficiently large [30]. It was demonstrated in [21] that the CRB on the sum of the MSEs for the target location estimation in an MIMO radar can be expressed as

$$
\sigma_{x, y}^{2}(\boldsymbol{p})=\frac{\boldsymbol{b}^{T} \boldsymbol{p}}{\boldsymbol{p}^{T} \boldsymbol{A} \boldsymbol{p}},
$$

where $\boldsymbol{p}$ is a vector denoting the UAV transmission powers, $\boldsymbol{b}=\left(\boldsymbol{g}_{a}+\boldsymbol{g}_{b}\right), \boldsymbol{A}=\boldsymbol{g}_{a} \boldsymbol{g}_{b}^{T}-\boldsymbol{g}_{c} \boldsymbol{g}_{c}^{T}, \boldsymbol{g}_{a}=\left[g_{a_{1}}, g_{a_{2}}, \cdots, g_{a_{K}}\right]^{T}$, $\boldsymbol{g}_{b}=\left[g_{b_{1}}, g_{b_{2}}, \cdots, g_{b_{K}}\right]^{T}, \boldsymbol{g}_{c}=\left[g_{c_{1}}, g_{c_{2}}, \cdots, g_{c_{K}}\right]^{T}$, with

$$
\begin{aligned}
& g_{a_{m}}=\xi \sum_{k=1}^{K}\left|\alpha_{m, k} l_{m, k}\right|^{2}\left(\frac{x_{m}-x_{t}}{R_{m, t}}+\frac{x_{k}-x_{t}}{R_{k, t}}\right)^{2} \\
& g_{b_{m}}=\xi \sum_{k=1}^{K}\left|\alpha_{m, k} l_{m, k}\right|^{2}\left(\frac{y_{m}-y_{t}}{R_{m, t}}+\frac{y_{k}-y_{t}}{R_{k, t}}\right)^{2}
\end{aligned}
$$

$$
\begin{aligned}
g_{c_{m}}= & \xi \sum_{k=1}^{K}\left|\alpha_{m, k} l_{m, k}\right|^{2}\left(\frac{x_{m}-x_{t}}{R_{m, t}}+\frac{x_{k}-x_{t}}{R_{k, t}}\right) \\
& \times\left(\frac{y_{m}-y_{t}}{R_{m, t}}+\frac{y_{k}-y_{t}}{R_{k, t}}\right) .
\end{aligned}
$$

In the above equations, $K$ is the number of UAVs, $\xi=$ $\frac{8 \pi^{2} B^{2}}{\sigma_{w}^{2} c^{2}}$, and $R_{m, t}$ is the distance from UAV $m$ to the center of search cell $\left(x_{t}, y_{t}\right)$. To achieve an accurate localization, the localization CRB needs to be smaller than a given localization accuracy threshold $\eta_{\max }$, which can be formulated as

$$
\sigma_{x, y}^{2}(\boldsymbol{p}) \leq \eta_{\max }
$$

Note that the UAV transmission power affects both the communication and radar sensing performance. Therefore, we need to carefully design the UAV transmission power.

\section{PROBLEM Formulation}

In this section, we first formulate the joint UAV locations, user association, and UAV transmission power optimization problem. The optimization problem is shown to be NP-hard, and hence it cannot be solved efficiently. We then propose to solve the problem with a method composed of two steps: 1) we apply the spectral clustering to cluster the ground users into several groups and determine the UAV locations accordingly, and the clustering result also provides an initial user association strategy; 2) we then solve the derived joint user association and UAV transmission power optimization via decomposing the problem into two sub-problems, and solve them iteratively.

\section{A. Basic Problem Formulation}

By assuming that the locations of the ground users are known, our goal is to maximize the network utility under the localization CRB constraint by jointly optimizing the UAV locations (i.e., $\boldsymbol{x}$ and $\boldsymbol{y}$ ), user association (i.e., $\boldsymbol{\Lambda}$ ), and UAV transmission power (i.e., $\boldsymbol{p}$ ). The optimization problem is formulated as

$$
\begin{aligned}
\max _{\boldsymbol{x}, \boldsymbol{y}, \boldsymbol{\Lambda}, \boldsymbol{p}} & \Sigma_{u}=\sum_{n \in \mathcal{N}} \log \left(\sum_{k \in \mathcal{K}} \lambda_{k, n} R_{k, n}\right) \\
\text { s.t. } & p_{\min } \leq p_{k} \leq p_{\max }, \quad \forall k \in \mathcal{K} \\
& \lambda_{k, n} \in\{0,1\}, \quad \forall n \in \mathcal{N}, \quad \forall k \in \mathcal{K}, \\
& \sum_{k=1}^{K} \lambda_{k, n}=1, \quad \forall n \in \mathcal{N} \\
& \sigma_{x, y}^{2}(\boldsymbol{p}) \leq \eta_{\text {max }},
\end{aligned}
$$

where $\Sigma_{u}$ denotes the network utility, and the constraint (14a) limits the UAV transmission power. Note that the minimum power $p_{\min }$ is set as a positive number to guarantee that each UAV is able to serve the users as well as locating the target.

Problem (14) is challenging to solve due to the following two main reasons. On one hand, the optimization variable $\boldsymbol{\Lambda}$ for user association is binary, i.e., (14b) and (14c) involve integer constraints. On the other hand, even with a fixed user association, the problem is still non-convex with respect to the UAV locations $\boldsymbol{x}, \boldsymbol{y}$ and/or UAV transmission power 
variable $\boldsymbol{p}$. Therefore, (14) is a mixed-integer non-convex problem, which is difficult to solve in general. Note that the optimal solution is hard to obtain even via exhaustive searching, since the UAV locations and transmission power are continuous variables, and the problem is non-convex with respect to them. To address such a challenging problem, we propose to decompose the original problem into three subproblems and optimize them in a sequential way. Although the three subproblems are not equivalent to the original one, the decomposition leads to sub-optimal but effective solutions to the originally intractable problem.

\section{B. Problem Decomposition}

To solve the problem (14) efficiently, we first determine the UAV locations by applying the spectral clustering mechanism [32] to cluster ground users into several groups according to their location relations. The location of each UAV is determined according to its associated user group. Subsequently, we decompose the derived joint user association and UAV transmission power optimization problem into two subproblems, i.e., user association sub-problem and UAV transmission power control sub-problem. In the user association sub-problem, the UAV transmission power is considered to be fixed. This sub-problem can be written as

$$
\begin{array}{ll}
\max _{\Lambda} & \Sigma_{u}=\sum_{n \in \mathcal{N}} \log \left(\sum_{k \in \mathcal{K}} \lambda_{k, n} R_{k, n}\right) \\
\text { s.t. } & \lambda_{k, n} \in\{0,1\}, \quad \forall n \in \mathcal{N}, \quad \forall k \in \mathcal{K}, \\
& \sum_{k=1}^{K} \lambda_{k, n}=1, \quad \forall n \in \mathcal{N} .
\end{array}
$$

With the user association indicator matrix $\boldsymbol{\Lambda}^{\star}$ being determined, the UAV transmission power control sub-problem can be expressed as

$$
\begin{array}{cl}
\max _{\boldsymbol{p}} & \Sigma_{u}=\sum_{n \in \mathcal{N}} \log \left(\sum_{k \in \mathcal{K}} \lambda_{k, n}^{\star} R_{k, n}\right) \\
\text { s.t. } & p_{\min } \leq p_{k} \leq p_{\max }, \quad \forall k \in \mathcal{K} \\
& \sigma_{x, y}^{2}(\boldsymbol{p}) \leq \eta_{\max } .
\end{array}
$$

\section{Proposed Algorithm}

In this section, we solve the optimization problem (14) and determine the UAV locations, user association, and UAV transmission power such that the network utility can be maximized under the constraint of the required localization CRB. First, we propose a cluster-based mechanism to adaptively divide users into different groups, based on which the UAV locations are determined. Subsequently, we iteratively solve the subproblem (15) and (16) to determine the user association and UAV transmission power. Specifically, we model the user association sub-problem (15) as a coalition game, while a SCA-based algorithm is presented to iteratively solve the UAV transmission power optimization sub-problem (16).

\section{A. UAV Locations and Initial User Association Determination}

As can be readily seen from (14), optimizing the UAV locations to maximize the total network utility is an NPhard problem. We propose to utilize the spectral clustering mechanism to determine the UAV locations. Since the communication links from the UAV to the ground users are dominated by the LoS links, the key factor that impacts the co-channel interference is physical distance separation of ground users. Therefore, we utilize the location similarities to group ground users based on the spectral clustering algorithm [32].

Algorithm 1 Spectral Clustering for Clustering Ground Users and Determining UAV Locations

Input: Number $K$ of clusters to construct, number $N$ of ground users, locations $\boldsymbol{u}, \boldsymbol{v}$ of $N$ ground users.

1: Compute the Gaussian distance similarity matrix $S$ using (17).

2: Compute the diagonal degree matrix $\boldsymbol{H}$ with diagonal elements $\left(d_{1}, \cdots, d_{N}\right)$, where $d_{i}=\sum_{j=1}^{N} s_{i, j}$.

3: Compute the normalized Laplacian matrix $L_{\text {sym }}=$ $\boldsymbol{H}^{-1 / 2}(\boldsymbol{H}-\boldsymbol{S}) \boldsymbol{H}^{-1 / 2}$.

4: Find the $K$ eigenvectors $\boldsymbol{e}_{1}, \cdots, \boldsymbol{e}_{K}$ of $L_{\text {sym }}$ corresponding to the $K$ smallest eigenvalues.

5: Let $\boldsymbol{U} \in \mathbb{R}^{N \times K}$ be the matrix containing the vectors $\boldsymbol{e}_{1}, \cdots, \boldsymbol{e}_{K}$ as columns.

6: Form the matrix $\boldsymbol{T} \in \mathbb{R}^{N \times K}$ from $\boldsymbol{U}$ by normalizing the rows to norm 1, i.e., $t_{i, j}=u_{i, j} / \sqrt{\sum_{k} u_{i, k}^{2}}$.

7: For $i=1, \cdots, N$, let $\boldsymbol{o}_{i} \in \mathbb{R}^{K}$ be the vector corresponding to the $i$-th row of $\boldsymbol{T}$.

8: Cluster $\left(\boldsymbol{o}_{i}\right)_{i=1, \cdots, N}$ with the $k$-means algorithm into clusters $C_{1}, \cdots, C_{K}$.

9: Determine the UAV locations as the centers of the clusters.

Output: Locations $\boldsymbol{x}, \boldsymbol{y}$ of $K$ UAVs, initial user association results.

To find locally coupled users in terms of distance, let $S$ denote the Gaussian distance similarity matrix, representing the similarities among ground users based on their geographical locations. The $\left(n_{1}, n_{2}\right)$-th element of the matrix $\boldsymbol{S}, s_{n_{1}, n_{2}}$, denotes the similarity among user $n_{1}$ and user $n_{2}$ in terms of their geographical locations, and is calculated as follows [33]

$$
s_{n_{1}, n_{2}}=\exp \left(-\frac{\left(u_{n_{1}}-u_{n_{2}}\right)^{2}+\left(v_{n_{1}}-v_{n_{2}}\right)^{2}}{2 \sigma_{d}^{2}}\right),
$$

where the parameter $\sigma_{d}$ controls the impact of neighborhood size. The rationale in (17) reflects that the distance similarity increases as the users come closer to each other and are more likely to be served by one UAV. We then utilize the spectral clustering algorithm to form clusters of ground users based on their Gaussian distance similarity matrix and determine the locations of UAVs based on the centers of the obtained clusters, as specified in Algorithm 1.

An example of the UAV locations and initial user association result is shown in Fig. 3. Conventionally, the UAVs are uniformly placed in the whole region, and the users are associated with the UAV which corresponds to the maximum SNR, i.e., the UAV which is the closest to them. As can be 

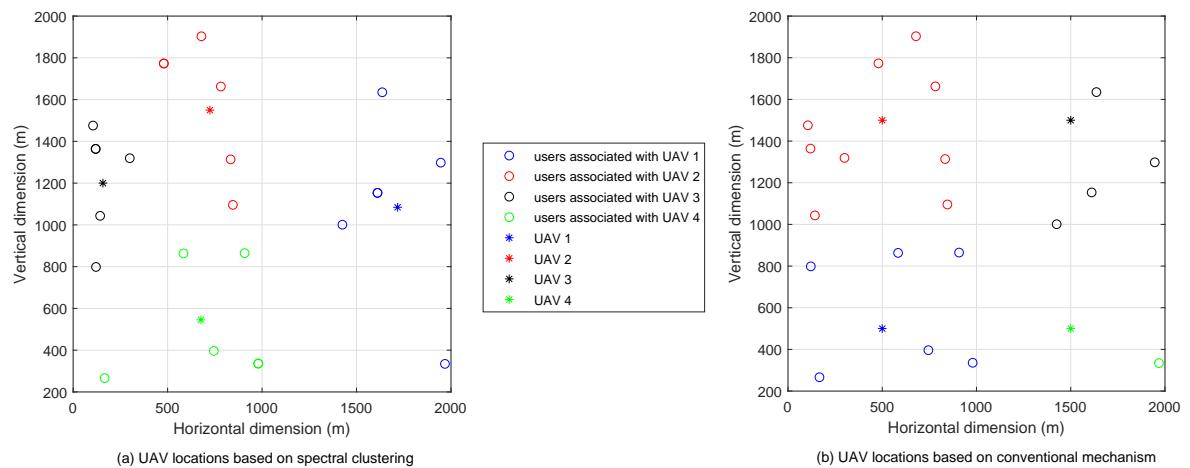

Fig. 3. An example of the UAV locations and initial user association based on spectral clustering and conventional mechanism

TABLE I

ACHIEVED NETWORK UTILITY AND TOTAL DATA RATE WITH DIFFERENT UAV LOCATION DETERMINATION METHODS.

\begin{tabular}{|c|c|c|}
\hline & $\begin{array}{c}\text { spectral clustering } \\
\text { based mechanism }\end{array}$ & $\begin{array}{c}\text { conventional } \\
\text { mechanism }\end{array}$ \\
\hline Achieved network utility & 336.29 & 323.84 \\
\hline Achieved data rate $(\mathrm{Mb} / \mathrm{s})$ & 498.6 & 233.5 \\
\hline
\end{tabular}

seen from Fig. 3(a), for the clustering-based mechanism, the numbers of users associated with different UAVs are more uniform, i.e., the load of each UAV is more balanced such that the bandwidths allocated to each user are more balanced, thus enhancing the fairness among different users, while for the conventional mechanism, as shown in Fig. 3(b), the load of each UAV is quite imbalanced. The achieved network utility and total rate of all users are shown in Table I. As can be seen, the performance of spectral clustering based mechanism outperforms that of conventional fixed mechanism.

\section{B. User Association based on Coalition Game}

For any given UAV transmission power $p$, the user association sub-problem (15) is a binary integer programming problem, and is hard to solve. A straightforward and efficient method is to assign users to the UAV associated with the maximum SNR [48]. However, according to (6), the effective rate is also affected by the choice of other users. Therefore, we formulate a coalition game [34] to handle the user association sub-problem.

Definition 1. (Coalition Game:) Let $\theta_{k}$ denote the coalition $k$, i.e., the set of users associated with $U A V k$, and $U_{k, n}$ denote the utility of user $n$ in coalition $\theta_{k}$. The coalition game is then defined as $(\Theta, U, \mathcal{K}, \mathcal{N})$, where $\Theta=\left\{\theta_{1}, \theta_{2}, \cdots, \theta_{K}\right\}$ is the coalition set and $U$ is the set of utilities. The utility of UAV $k$ is defined as

$$
U_{k}\left(\theta_{k}\right)=\sum_{n \in \theta_{k}} \log \left(R_{k, n}\right) .
$$

Since the UAVs serving a small number of users can spare more resources, by transferring some users from heavy-loaded UAVs to light-loaded UAVs, the utility of these users can be improved, thus potentially improving the whole network utility. Based on the utilitarian order in the coalition formation game model [35], we introduce the rule for transferring the user $n$ from coalition $\theta_{i}$ to $\theta_{j}$ as follows [12]

$$
\begin{aligned}
& R_{j, n} \geq R_{i, n}, \\
& U_{i}\left(\theta_{i}^{\prime}\right)+U_{j}\left(\theta_{j}^{\prime}\right)>U_{i}\left(\theta_{i}\right)+U_{j}\left(\theta_{j}\right),
\end{aligned}
$$

where $\theta_{i}^{\prime}$ and $\theta_{j}^{\prime}$ represent the coalition after the transferring. The first condition guarantees that the utility of user $n$ is not decreased, and the second condition guarantees that the total utility of network is improved. In case there are multiple users satisfying the transferring rule, the user to be transferred is selected so that the total network utility is maximized.

Theorem 1. Under the utilitarian order, the constructed coalition game reaches a stable coalition structure.

Proof: It can be seen from the system model that the number of game participants is finite in the constructed coalition game, thus the number of possible transfers is limited. In addition, in the transferring process, the total network utility is monotonically increased. Therefore, the coalition game is guaranteed to converge to a coalition equilibrium structure. A more detailed demonstration process can be found in [36].

\section{UAV transmission power Control based on Successive Convex Approximation}

For any given UAV locations as well as user association $\{\boldsymbol{\Lambda}, \boldsymbol{x}, \boldsymbol{y}\}$, the UAV transmission power control sub-problem (16) is non-convex. To simplify the objective function, we introduce an auxiliary variable $\chi=\left\{\chi_{1}, \cdots, \chi_{N}\right\}$, and the sub-problem (16) can be reformulated as

$$
\begin{array}{ll}
\max _{\boldsymbol{p}, \boldsymbol{\chi}} & \sum_{n} \log \left(\chi_{n}\right) \\
\text { s.t. } & \chi_{n} \leq B \sum_{k} \rho_{k, n} \log _{2}\left(1+\Gamma_{k, n}\right), \\
& \sigma_{x, y}^{2}(\boldsymbol{p}) \leq \eta_{\max }, \\
& p_{\min } \leq p_{k} \leq p_{\max },
\end{array}
$$

where $\rho_{k, n}=\lambda_{k, n} / \beta_{k}$. However, problem (21) is still a nonconvex optimization problem due to the non-convex constraint (21a), and it is in fact NP-hard for a general $N$. To handle the non-convex constraint (21a), we apply the successive convex approximation technique [37]. 
First, we introduce the auxiliary variable $p_{k}=e^{q_{k}}$ and relax the constraint (21b) as in [21]. Thus, the problem (21) is transformed as follows:

$$
\begin{array}{cl}
\max _{\boldsymbol{q}, \boldsymbol{\chi}} & \sum_{n} \log \left(\chi_{n}\right) \\
\text { s.t. } & \chi_{n} \leq B \sum_{k} \rho_{k, n} \log _{2}\left(1+\tilde{\Gamma}_{k, n}\right), \\
& \boldsymbol{b}-\eta_{\max } \boldsymbol{A} \tilde{\boldsymbol{q}} \leq \mathbf{0} \\
& \log \left(p_{\min }\right) \leq q_{k} \leq \log \left(p_{\max }\right)
\end{array}
$$

where $\tilde{q}=\left[e^{q_{1}}, e^{q_{2}}, \cdots, e^{q_{K}}\right]^{T}$, and the intermediate variable $\tilde{\Gamma}_{k, n}$ is given by

$$
\tilde{\Gamma}_{k, n}=\frac{e^{q_{k}} h_{k, n}^{2}}{\sum_{k^{\prime} \in \mathcal{K} \backslash k} e^{q_{k^{\prime}}} h_{k^{\prime}, n}^{2}+N_{0}} .
$$

Following the development of (19) of [38], we have the following inequality

$$
\log (1+z) \geq \zeta \log (z)+\phi
$$

which is tight at $z=z_{0}$ when the approximation constants are chosen as

$$
\begin{aligned}
\zeta & =\frac{z_{0}}{1+z_{0}}, \\
\phi & =\log \left(1+z_{0}\right)-\frac{z_{0}}{1+z_{0}} \log \left(z_{0}\right) .
\end{aligned}
$$

By introducing superscripts $(l)$ and $(l+1)$ to represent the value of variables at the $l$ th and $(l+1)$ th iteration, we obtain the following lower bound for $\log _{2}\left(1+\Gamma_{k, n}^{(l+1)}\right)$, i.e.,

$$
\log _{2}\left(1+\tilde{\Gamma}_{k, n}^{(l+1)}\right) \geq \zeta_{k, n}^{(l)} \log _{2}\left(\tilde{\Gamma}_{k, n}^{(l+1)}\right)+\phi_{k, n}^{(l)}
$$

where $\zeta_{k, n}=\frac{\tilde{\Gamma}_{k, n}^{(l)}}{1+\tilde{\Gamma}_{k, n}^{(l)}}$ and $\phi_{k, n}=\log _{2}\left(1+\tilde{\Gamma}_{\mathrm{k}, \mathrm{n}}^{(1)}\right)-$ $\frac{\tilde{\Gamma}_{k, n}^{(l)}}{1+\tilde{\Gamma}_{k, n}^{(l)}} \log _{2}\left(\tilde{\Gamma}_{k, n}^{(l)}\right)$. Similarly, by applying first-order Taylor series expansions on $e^{q_{k}}$, we have $e^{q_{k}^{(l)}}\left(q_{k}^{(l+1)}-q_{k}^{(l)}+1\right) \leq$ $e^{q_{k}^{(l+1)}}$

Therefore, the problem (22) is approximated as the following problem

$$
\begin{aligned}
& \max _{\boldsymbol{q}^{(l+1)}, \boldsymbol{\chi}^{(l+1)}} \sum_{n} \log \left(\chi_{n}^{(l+1)}\right) \\
& \text { s.t. } \quad \chi_{n}^{(l+1)} \leq \sum_{k} B \rho_{k, n} \zeta_{k, n}^{(l)} \log _{2}\left(\tilde{\Gamma}_{k, n}^{(l+1)}\right) \\
& +\sum_{k} B \rho_{k, n} \phi_{k, n}^{(l)}, \\
& \boldsymbol{b} \leq \eta_{\max } \boldsymbol{A} \hat{\boldsymbol{q}}^{(\boldsymbol{l + 1})}, \\
& \log \left(p_{\min }\right) \leq q_{k} \leq \log \left(p_{\max }\right),
\end{aligned}
$$

where $\hat{\boldsymbol{q}}^{(\boldsymbol{l + 1})}=\left[e^{q_{1}^{(l)}}\left(q_{1}^{(l+1)}-q_{1}^{(l)}+1\right), \cdots, e^{q_{K}^{(l)}}\left(q_{K}^{(l+1)}-\right.\right.$ $\left.\left.q_{K}^{(l)}+1\right)\right]^{T}$.

Theorem 2. Problem (28) is a convex optimization problem.

Proof: It can be readily seen that the constraints (28b) and (28c) are convex. Therefore, we focus on the convexity of constraint (28a). Note that $\log _{2}\left(\tilde{\Gamma}_{k, n}^{(l+1)}\right)$ can be expressed as

$$
\begin{aligned}
\log _{2}\left(\tilde{\Gamma}_{k, n}^{(l+1)}\right)= & \frac{1}{\log (2)}\left[\log \left(h_{k, n}^{2}\right)+q_{k}-\right. \\
& \left.\log \left(\sum_{k^{\prime} \in \mathcal{K} \backslash k} e^{q_{k^{\prime}}} h_{k^{\prime}, n}^{2}+N_{0}\right)\right] ;
\end{aligned}
$$

thus, $\log _{2}\left(\tilde{\Gamma}_{k, n}^{(l+1)}\right)$ is a concave function since the log-sumexponential function is convex [39]. Meanwhile, the objective function (28) is concave, because it is a combination of multiple concave functions. Therefore, problem (28) is a convex optimization problem.

Thus, the UAV transmission power control sub-problem (16) can be solved via the SCA algorithm.

\section{Overall Algorithm and Convergence}

Based on the algorithms presented in the previous three subsections, we present an overall algorithm for solving problem (14). Specifically, we first utilize the spectral clustering mechanism to cluster the group users in order to determine the locations of UAVs and the initial user association schemes. Then, the user association $\Lambda$ and UAV transmission power $\boldsymbol{p}$ are alternately optimized by solving sub-problem (15) and (16) correspondingly, while keeping the other variable fixed. Furthermore, the obtained solution in each iteration is used as the input of the next iteration. The details of the overall algorithm is summarized in Algorithm 2.

In what follows, we prove the convergence of the overall algorithm.

\section{Theorem 3. The proposed overall algorithm is convergent.}

Proof: First, we consider user association in the $(l+1)$ th iteration. Note that the user association and its corresponding network utility will not be updated unless the network utility obtained in the coalition game is larger than the current network utility. Therefore, we have

$$
U\left(\boldsymbol{\Lambda}^{(l+1)}, \boldsymbol{p}^{(l)}\right) \geq U\left(\boldsymbol{\Lambda}^{(l)}, \boldsymbol{p}^{(l)}\right),
$$

where $U(\cdot)$ denotes the network utility with the corresponding parameters.

When solving the UAV transmission power control subproblem, the optimal power control $\boldsymbol{p}^{(l+1)}$ with $\boldsymbol{C}^{(l+1)}$ is asymptotically obtained via SCA. Thus we have

$$
U\left(\boldsymbol{\Lambda}^{(l+1)}, \boldsymbol{p}^{(l+1)}\right) \geq U\left(\boldsymbol{\Lambda}^{(l+1)}, \boldsymbol{p}^{(l)}\right),
$$

Therefore, in the $(l+1)$ th iteration, we have

$$
U\left(\boldsymbol{\Lambda}^{(l+1)}, \boldsymbol{p}^{(l+1)}\right) \geq U\left(\boldsymbol{\Lambda}^{(l+1)}, \boldsymbol{p}^{(l)}\right) \geq U\left(\boldsymbol{\Lambda}^{(l)}, \boldsymbol{p}^{(l)}\right),
$$

i.e., the objective function is non-decreasing. Also note that the network utility is upper-bounded by a finite value. Therefore, the overall algorithm is convergent.

Remark 3. The computational complexity for solving the problem (14) based on Algorithm 2 is briefly discussed here. It mainly consists of three parts. Specifically, the complexity for 


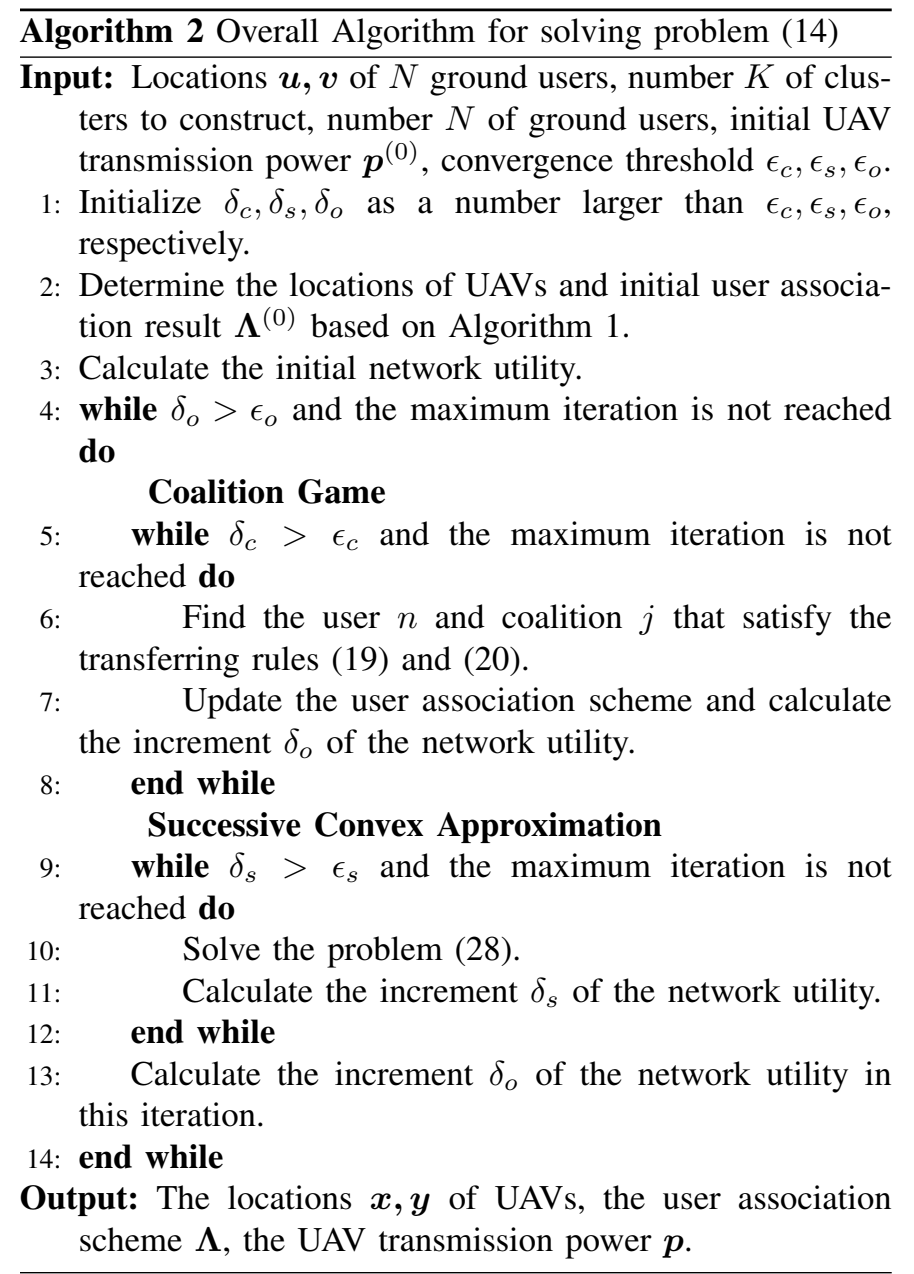

the spectral clustering based UAV location determination is $O(K N)$ [32]. For the coalition game based user association, the complexity in each iteration is $O(K N)$ [12]; thus, the complexity of user association is $O(L K N)$, where $L$ is the number of iterations. For the SCA based transmission power control, as the approximated problem (28) is convex, the complexity is polynomial over $(K+N)$. To conclude, the total computational complexity is polynomial over $K N$.

\section{Simulation Results}

In this section, we evaluate the performance of the proposed algorithms. The locations of the ground users are randomly and uniformly distributed in a 2-dimension area of $2 \mathrm{~km} \times 2$ $\mathrm{km}$. The simulation parameters are listed in Table II, where the values of parameters related to UAV, i.e., the UAV altitude, noise power, maximum transmission power, are selected according to [11], and the channel power gain in [11] is also adopted. The OFDM-based DFRC signals proposed in [44] are adopted, and the bandwidth is set as $51.2 \mathrm{MHz}$. The magnitude of the RCS $\alpha$ is assumed to be uniformly distributed between 0.8 to 1 . Without loss of generality, the center of the extended target is assumed to be $\left(x_{t}, y_{t}\right)=(1000 \mathrm{~m}, 1000 \mathrm{~m})$. To our best knowledge, there has been no similar work reported where the distributed DFRC system is considered. Therefore, we compare our scheme with a conventional and general scheme
TABLE II

SimULATION PARAMETERS

\begin{tabular}{|c|c|}
\hline Center frequency & $35 \mathrm{GHz}[14]$ \\
\hline UAV altitude $H$ & $100 \mathrm{~m} \mathrm{[11]}$ \\
\hline Noise power $N_{0}$ & $-110 \mathrm{dBm}[11]$ \\
\hline Channel power gain at reference distance $\rho_{0}$ & $-60 \mathrm{~dB}[11]$ \\
\hline Bandwidth of each sub-channel & $51.2 \mathrm{MHz}[44]$ \\
\hline Maximum UAV transmission power & $20 \mathrm{dBm} \mathrm{[11]}$ \\
\hline Minimum UAV transmission power & $0 \mathrm{dBm}$ \\
\hline Localization accuracy threshold $\eta_{m a x}$ & $25 \mathrm{~cm}^{2}$ \\
\hline Impact of neighborhood size $\sigma_{d}$ & 300 \\
\hline Coalition game convergence threshold $\epsilon_{c}, \epsilon_{s}, \epsilon_{o}$ & 0.01 \\
\hline Maximum iteration number & 10 \\
\hline Default number of UAVs & 4 \\
\hline Default number of ground users & 20 \\
\hline
\end{tabular}

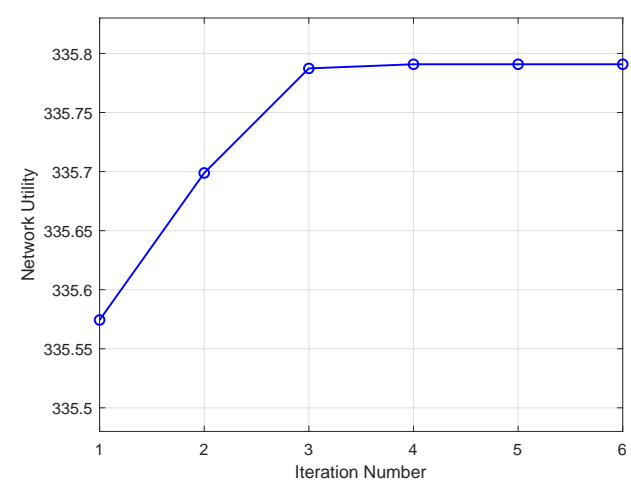

Fig. 4. Total network utility v.s. iteration numbers $(K=4, N=20)$.

where the locations of UAVs are fixed in a uniform way. In addition, we also compare our scheme with the joint UAV locations and user association optimization design in [48] to show the effectiveness of the proposed algorithm. Specifically, the performance of the following four algorithms are compared with each other:

(1) "Fixed location + Fixed power": the locations of UAVs are uniformly distributed in the whole area. The users are associated with the nearest UAV, and the UAV transmission power is set at the maximum;

(2) "Dynamic location + Fixed power": the locations of UAVs and the user association schemes are determined based on Algorithm 1, and the UAV transmission power is set at the maximum;

(3) "Dynamic location + Dynamic power": the locations of UAVs, the user association schemes, and the UAV transmission power are determined based on Algorithm 2.

(4) "[48] + Max power": the locations of UAVs and the user association schemes are determined based on the algorithm proposed in [48], while the UAV transmission power is set at the maximum to fulfill the requirement of localization.

Before performance comparison, we show the convergence behaviour of Algorithm 2 in Fig. 4. It can be observed that the total network utility achieved by Algorithm 2 is able to converge in only a few iterations.

\section{A. Performance with Various Numbers of UAVs}

Fig. 5(a) depicts the total network utility under various numbers of UAVs. As can be seen, the network utilities of 

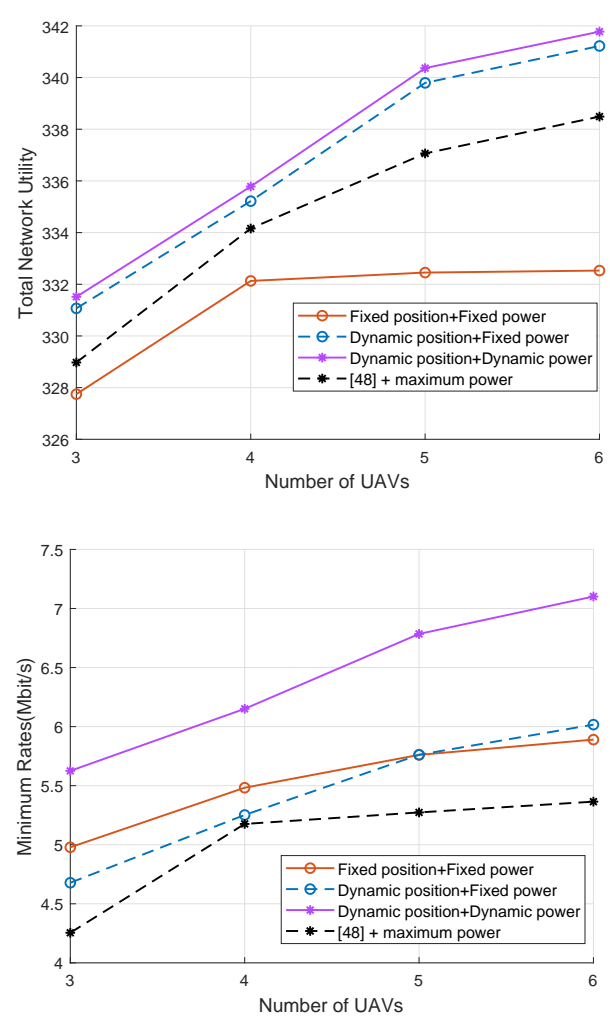

Fig. 5. (a) Total network utility v.s. number of UAVs $(N=20)$. (b) Minimum user data rate v.s. number of UAVs $(N=20)$.

all four schemes increase with UAVs due to the increase of available resources. For the "Fixed position + Fixed power" scheme, the increasing speed of the network utility decreases with the increase of the number of UAVs. This is because the co-channel interference increases with the number of UAVs, especially for the case of fixed position and fixed power. While for the schemes in which the UAV locations are dynamically determined by Algorithm 1 and the algorithm proposed in [48], the network utility increases more quickly. Moreover, the network utility of dynamic power scheme outperforms that of fixed power scheme since the co-channel interference problem is addressed via power control.

In network utility maximization problem, one issue that needs to be considered is the fairness, and the minimum user data rate can also reflect the fairness of the network. In Fig. 5(b), we show the minimum user data rate under various numbers of UAVs. Again, the minimum user data rates of all four schemes increase with the number of UAVs, and the schemes with UAV locations being dynamically determined outperforms that with fixed position. As the number of UAVs increases, the gap between the "Dynamic position + Dynamic power" scheme and "Dynamic position + Fixed power" scheme increases, which indicates the proposed algorithm can improve the user fairness in the whole network. This is because for a larger number of UAVs, power control is more desirable to deal with the increasing co-channel interference. However, the minimum user rate will not increase boundlessly, and it will converge to a point where the balance of the adjusted transmission power and number of UAVs is achieved. Note that
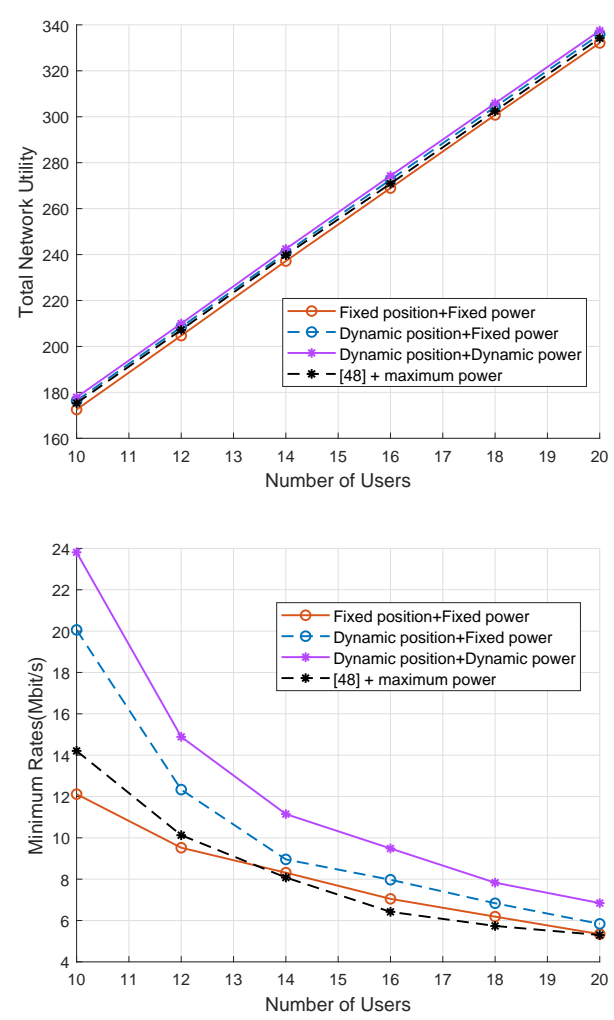

Fig. 6. (a) Total network utility v.s. number of ground users $(K=4)$. (b) Minimum user rate v.s. number of ground users $(K=4)$.

although the network utility of the scheme in [48] outperforms that of the "Fixed position + Fixed power" scheme, the minimum user rate of the scheme in [48] is the lowest.

In Table III, the average transmission power per UAV as well as the average CRB for localization under various numbers of UAVs are shown. With the number of UAVs increasing, the CRBs of all three schemes decrease due to the increasing diversity gain of cooperative detection, and the transmission power in the dynamic power scheme is decreased to reduce the co-channel interference. Due to the reduction of transmission power, the dynamic power scheme has slightly larger CRB than the fixed power schemes. Meanwhile, the CRBs of the four schemes are comparable, and they all satisfy the localization accuracy constraint.

\section{B. Performance with Various Numbers of Ground Users}

Fig. 6(a) depicts the total network utility under various numbers of ground users. As can be seen, the network utilities of all four schemes increase almost proportionally with the number of UAVs due to the increase of available resources, which is consistent with the calculation of the total network utility. Also, the performance of the dynamic power scheme outperforms that of the fixed power schemes, and the dynamic UAV location schemes outperform the fixed location one.

In Fig. 6(b), we show the minimum user data rate under various numbers of ground users. As can be seen, the minimum user rate decreases with the number of ground users increasing due to the increase of the load for each UAV; thus, 
TABLE III

AVERAGE TRANSMISSION POWER PER UAV (MW) / AVERAGE ACHIEVED CRB FOR LOCALIZATION $\left(\mathrm{cm}^{2}\right)$.

\begin{tabular}{|c|c|c|c|c|}
\hline $\begin{array}{c}\text { Number of } \\
\text { UAVs }\end{array}$ & $\begin{array}{c}\text { Fixed location } \\
\text { Fixed power }\end{array}$ & $\begin{array}{c}\text { Dynamic location } \\
\text { Fixed power }\end{array}$ & $\begin{array}{c}\text { Dynamic location } \\
\text { Dynamic power }\end{array}$ & $\begin{array}{c}{[48]} \\
\text { Max power }\end{array}$ \\
\hline 3 & $100 / 22.0$ & $100 / 12.0$ & $85.5 / 13.0$ & $100 / 18.0$ \\
\hline 4 & $100 / 17.0$ & $100 / 8.0$ & $82.8 / 9.8$ & $100 / 17.0$ \\
\hline 5 & $100 / 7.3$ & $100 / 6.6$ & $80.8 / 8.3$ & $100 / 5.9$ \\
\hline 6 & $100 / 5.6$ & $100 / 3.8$ & $81.0 / 5.1$ & $100 / 4.8$ \\
\hline
\end{tabular}

TABLE IV

AVERAGE TRANSMission POWER PER UAV (MW)/ AVERAGE ACHIEVED CRB FOR LOCALIZATION $\left(\mathrm{cm}^{2}\right)$.

\begin{tabular}{|c|c|c|c|c|}
\hline $\begin{array}{c}\text { Number of } \\
\text { ground users }\end{array}$ & $\begin{array}{c}\text { Fixed location } \\
\text { Fixed power }\end{array}$ & $\begin{array}{c}\text { Dynamic location } \\
\text { Fixed power }\end{array}$ & $\begin{array}{c}\text { Dynamic location } \\
\text { Dynamic power }\end{array}$ & $\begin{array}{c}{[48]} \\
\text { Max power }\end{array}$ \\
\hline 10 & $100 / 17.0$ & $100 / 7.3$ & $78.2 / 9.7$ & $100 / 18.0$ \\
\hline 12 & $100 / 17.0$ & $100 / 6.4$ & $78.1 / 8.5$ & $100 / 18.0$ \\
\hline 14 & $100 / 17.0$ & $100 / 7.5$ & $79.0 / 9.5$ & $100 / 17.0$ \\
\hline 16 & $100 / 17.0$ & $100 / 8.0$ & $80.8 / 9.8$ & $100 / 17.0$ \\
\hline 18 & $100 / 17.0$ & $100 / 8.8$ & $84.7 / 10.0$ & $100 / 17.0$ \\
\hline 20 & $100 / 17.0$ & $100 / 8.3$ & $84.5 / 10.0$ & $100 / 17.0$ \\
\hline
\end{tabular}

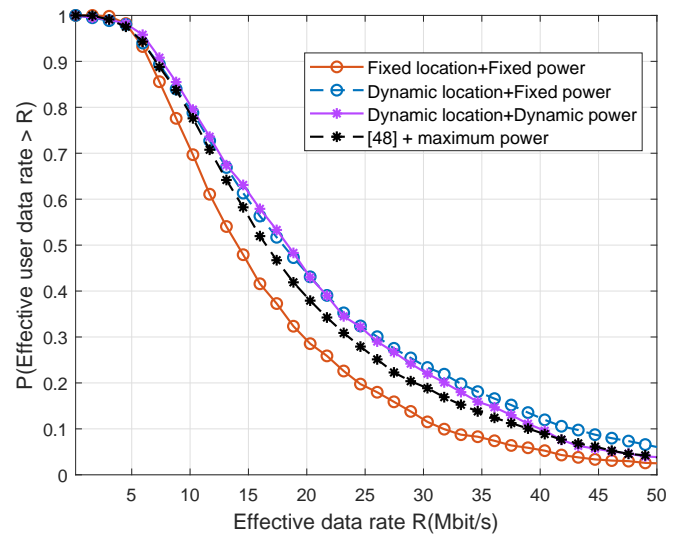

Fig. 7. The CCDF curves of the user rates $(N=20, K=4)$.

each user is allocated with a smaller bandwidth. Meanwhile, the gap between fixed position scheme and dynamic position scheme decreases with the number of ground users increasing. This is because as the number of users increases, the diversity gain between users and UAVs increases. And it is more likely that the users are uniformly distributed in the whole region; thus, the positions of UAVs play a less important role.

In Table IV, the average transmission power per UAV as well as average achieved CRB for localization under various numbers of ground users are shown. Again, it is shown that the "Dynamic location Dynamic power" scheme maintains comparable localization accuracy with the "Dynamic location + Fixed power" scheme with less transmission power consumption.

\section{Complementary Cumulative Distribution Function of the Minimum Data Rate}

In Fig. 7, we show the complementary cumulative distribution function (CCDF) of the achieved user data rate of the four schemes. It is shown that the user rates of the dynamic UAV location schemes are significantly larger than those of the fixed UAV location scheme, i.e., the proportion of users with moderate to high data rates is larger under dynamic UAV positions. Meanwhile, the low-rate users under "Dynamic position + Dynamic power" achieve higher data rate than those under "Dynamic position + Fixed power", while for the highrate users, the opposite situation happens, which indicates that the proportion of the users with moderate data rates is larger under dynamic power. This phenomenon also validates the effectiveness of the proposed SCA-based power control algorithm and reveals the importance of UAV transmission power control in improving the fairness in the whole network.

\section{Vi. Conclusion And Future Work}

In this paper, we studied joint UAV location, user association, and UAV transmission power control in a DFRC multi-UAV network, where multiple UAVs are employed to simultaneously serve a group of ground users for communications and cooperatively sense the targets. To address the cochannel interference and maximize the total network utility, we employed the techniques of spectral clustering, coalition game, and successive convex approximation to optimize the UAV location, user association and UAV transmission power, respectively. We then presented an overall algorithm to jointly optimize the three variables. Simulation results show that the proposed algorithm improves the minimum user data rate significantly and improves the fairness of the network. Moreover, the proposed algorithm improves the network utility, compared to conventional techniques.

The proposed system model and cooperative communication and radar sensing protocol of DFRC-MUN in this paper can be applied/extended to a class of DFRC-MUN optimization problems under some general setups and with other performance considerations, some of which are discussed as follows for future work.

- Joint spectrum and power allocation in DFRC-MUN: In this paper, all UAVs share the same frequency band, thus introducing interference to ground users. By dividing the total spectrum into different orthogonal bands and allocating them to different UAVs, the interference can be mitigated effectively, which can potentially improve 
the network utility and decrease the complexity in radar signal processing.

- Resource allocation in DFRC-MUN equipped with multiple antennas: In this paper, all UAVs are assumed to be equipped with one antenna. By deploying multiple antennas for UAVs, more design freedoms can be obtained; hence, not only multi-user interference can be controlled, but the localization accuracy can also be improved.

- UAV trajectory design in DFRC-MUN: In this paper, the locations of the UAVs are assumed to be fixed. By taking the UAV trajectory design into consideration, the network utility can be further improved. However, since the problem is highly non-convex with respect to UAV locations, it's challenging to solve it via conventional optimization technique, while reinforcement learning is a promising method.

\section{REFERENCES}

[1] B. Li, Z. Fei and Y. Zhang, "UAV Communications for 5G and beyond: Recent advances and future trends," IEEE Internet of Things Journal, vol. 6, no. 2, pp. 2241-2263, April 2019.

[2] Y. Zeng, J. Lyu and R. Zhang, "Cellular-connected UAV: potential, challenges, and promising technologies," IEEE Wireless Communications, vol. 26, no. 1, pp. 120-127, February 2019.

[3] A. Fotouhi et al., "Survey on UAV cellular communications: Practical aspects, standardization advancements, regulation, and security challenges," IEEE Communications Surveys \& Tutorials, vol. 21, no. 4, pp. 3417-3442, Fourthquarter 2019.

[4] B. Li, Z. Fei, Y. Dai and Y. Zhang, "Secrecy-optimized resource allocation for UAV-assisted relaying networks," in 2018 IEEE Global Communications Conference (GLOBECOM), Abu Dhabi, United Arab Emirates, 2018, pp. 1-6.

[5] A. Ryan, M. Zennaro, A. Howell, R. Sengupta and J. K. Hedrick, "An overview of emerging results in cooperative UAV control," in 2004 43rd IEEE Conference on Decision and Control (CDC) (IEEE Cat. No.04CH37601), Nassau, 2004, pp. 602-607 Vol.1.

[6] Y. Ji, C. Dong, X. Zhu and Q. Wu, "Fair-energy trajectory planning for cooperative UAVs to locate multiple targets," in ICC 2019 - 2019 IEEE International Conference on Communications (ICC), Shanghai, China, 2019, pp. 1-7.

[7] B. Li, Z. Fei, Y. Zhang and M. Guizani, "Secure UAV communication networks over 5G," IEEE Wireless Communications, vol. 26, no. 5, pp. 114-120, October 2019.

[8] Y. Zeng and R. Zhang, "Energy-efficient UAV communication with trajectory optimization," IEEE Transactions on Wireless Communications, vol. 16, no. 6, pp. 3747-3760, June 2017.

[9] G. Zhang, Q. Wu, M. Cui and R. Zhang, "Securing UAV communications via joint trajectory and power control," IEEE Transactions on Wireless Communications, vol. 18, no. 2, pp. 1376-1389, Feb. 2019.

[10] Q. Wu and R. Zhang, "Common throughput maximization in UAVenabled OFDMA systems with delay consideration," IEEE Transactions on Communications, vol. 66, no. 12, pp. 6614-6627, Dec. 2018.

[11] Q. Wu, Y. Zeng and R. Zhang, "Joint trajectory and communication design for multi-UAV enabled wireless networks," IEEE Transactions on Wireless Communications, vol. 17, no. 3, pp. 2109-2121, March 2018.

[12] M. Sami and J. N. Daigle, "User association and power control for UAVenabled cellular networks," IEEE Wireless Communications Letters, vol. 9, no. 3, pp. 267-270, March 2020.

[13] D. Mishra, P. Lohan and L. N. Devi, "Coverage-constrained utility maximization of UAV," in ICC 2019 - 2019 IEEE International Conference on Communications (ICC), Shanghai, China, 2019, pp. 1-6.

[14] Y. K. Kwag, M. S. Choi, C. H. Jung and K. Y. Hwang, "Collision avoidance radar for UAV," in 2006 CIE International Conference on Radar, Shanghai, 2006, pp. 1-4.

[15] Y. A. Nijsure, G. Kaddoum, N. Khaddaj Mallat, G. Gagnon and F. Gagnon, "Cognitive chaotic UWB-MIMO detect-avoid Radar for autonomous UAV navigation," IEEE Transactions on Intelligent Transportation Systems, vol. 17, no. 11, pp. 3121-3131, Nov. 2016.
[16] S. Xu, K. Doganay and H. Hmam, "Distributed path optimization of multiple UAVs for AOA target localization," in 2016 IEEE International Conference on Acoustics, Speech and Signal Processing (ICASSP), Shanghai, 2016, pp. 3141-3145.

[17] J. Gu, T. Su, Q. Wang, X. Du and M. Guizani, "Multiple moving targets surveillance based on a cooperative network for multi-UAV," IEEE Communications Magazine, vol. 56, no. 4, pp. 82-89, April 2018.

[18] A. Guerra, D. Dardari and P. M. Djuric, "Dynamic radar networks of UAVs: A tutorial overview and tracking performance comparison with terrestrial radar networks," IEEE Vehicular Technology Magazine, vol. 15, no. 2, pp. 113-120, June 2020.

[19] K. Shen and W. Yu, "Distributed pricing-based user association for downlink heterogeneous cellular networks," IEEE Journal on Selected Areas in Communications, vol. 32, no. 6, pp. 1100-1113, June 2014.

[20] Q. Ye, B. Rong, Y. Chen, M. Al-Shalash, C. Caramanis and J. G. Andrews, "User association for load balancing in heterogeneous cellular networks," IEEE Transactions on Wireless Communications, vol. 12, no. 6, pp. 2706-2716, June 2013.

[21] H. Godrich, A. P. Petropulu and H. V. Poor, "Power allocation strategies for target localization in distributed multiple-radar architectures," IEEE Transactions on Signal Processing, vol. 59, no. 7, pp. 3226-3240, July 2011.

[22] A. Hassanien, M. G. Amin, Y. D. Zhang and F. Ahmad, "Dual-function radar-communications: Information embedding using sidelobe control and waveform diversity," IEEE Transactions on Signal Processing, vol. 64, no. 8, pp. 2168-2181, April15, 2016.

[23] F. Liu, L. Zhou, C. Masouros, A. Li, W. Luo and A. Petropulu, "Toward dual-functional radar-communication systems: Optimal waveform design," IEEE Transactions on Signal Processing, vol. 66, no. 16, pp. 4264-4279, 15 Aug.15, 2018.

[24] F. Liu, L. Zhou, C. Masouros, A. Lit, W. Luo and A. Petropulu, "Dualfunctional cellular and radar transmission: Beyond coexistence," in 2018 IEEE 19th International Workshop on Signal Processing Advances in Wireless Communications (SPAWC), Kalamata, 2018, pp. 1-5.

[25] F. Liu, C. Masouros, A. Li, H. Sun and L. Hanzo, "MU-MIMO communications with MIMO radar: From co-existence to joint transmission," IEEE Transactions on Wireless Communications, vol. 17, no. 4, pp. 27552770, April 2018.

[26] C. Sturm, T. Zwick and W. Wiesbeck, "An OFDM system concept for joint radar and communications operations," in VTC Spring 2009 - IEEE 69th Vehicular Technology Conference, Barcelona, 2009, pp. 1-5.

[27] J. A. Zhang, X. Huang, Y. J. Guo, J. Yuan and R. W. Heath, "Multibeam for joint communication and radar sensing using steerable analog antenna arrays," IEEE Transactions on Vehicular Technology, vol. 68, no. 1, pp. 671-685, Jan. 2019.

[28] Y. Luo, J. A. Zhang, W. Ni, J. Pan and X. Huang, "Constrained multibeam optimization for joint communication and radio sensing," in 2019 IEEE Global Communications Conference (GLOBECOM), Waikoloa, HI, USA, 2019, pp. 1-6.

[29] M. I. Skolnik, Ed., Radar Handbook, 3rd ed. New York, NY, USA: McGraw-Hill, 2008.

[30] H.L.Van Trees and K. L. Bell, Bayesian Bounds for Parameter Estimation and Nonlinear Filtering/Tracking. New York: Wiley-Intersci., 2007.

[31] N. Merayo, P. Pavon-Marino, J. C. Aguado, R. J. Durn, F. Burrull and V. Bueno-Delgado, "Fair bandwidth allocation algorithm for PONS based on network utility maximization," IEEE/OSA Journal of Optical Communications and Networking, vol. 9, no. 1, pp. 75-86, Jan. 2017.

[32] U. Luxburg, "A tutorial on spectral clustering," Springer J. Stat. Comput., vol. 17, no. 4, pp. 395C416, Dec. 2007.

[33] M. I. Ashraf, M. Bennis, W. Saad, M. Katz and C. Hong, "Dynamic clustering and user association in wireless small-cell networks with social considerations," IEEE Transactions on Vehicular Technology, vol. 66, no. 7, pp. 6553-6568, July 2017.

[34] B. Zhang, X. Mao, J. Yu and Z. Han, "Resource allocation for 5G heterogeneous cloud radio access networks with D2D communication: A matching and coalition approach," IEEE Transactions on Vehicular Technology, vol. 67, no. 7, pp. 5883-5894, July 2018.

[35] W. Saad, Z. Han, M. Debbah, A. Hjorungnes and T. Basar, "Coalitional game theory for communication networks," IEEE Signal Processing Magazine, vol. 26, no. 5, pp. 77-97, September 2009.

[36] K. R. Apt and A. Witzel, "A generic approach to coalition formation," Int. Game Theory Rev., vol. 11, no. 3, pp. 347-367, 2009.

[37] Z. Fei, B. Li, S. Yang, C. Xing, H. Chen and L. Hanzo, "A survey of multi-objective optimization in wireless sensor networks: Metrics, algorithms, and open problems," IEEE Communications Surveys \& Tutorials, vol. 19, no. 1, pp. 550-586, Firstquarter 2017. 
[38] J. Papandriopoulos, S. Dey and J. Evans, "Optimal and distributed protocols for cross-layer design of physical and transport layers in MANETs," IEEE/ACM Transactions on Networking, vol. 16, no. 6, pp. 1392-1405, Dec. 2008.

[39] S. Boyd and L. Vandenberghe, Convex Optimization. New York, NY, USA: Cambridge University Press, 2004.

[40] X. Wang, A. Hassanien and M. G. Amin, "Dual-function MIMO radar communications system design via sparse array optimization," IEEE Transactions on Aerospace and Electronic Systems, vol. 55, no. 3, pp. 1213-1226, June 2019.

[41] C. Shi, F. Wang, S. Salous and J. Zhou, "Joint subcarrier assignment and power allocation strategy for integrated radar and communications system based on power minimization," IEEE Sensors Journal, vol. 19, no. 23, pp. 11167-11179, 1 Dec.1, 2019.

[42] C. Shi, F. Wang, M. Sellathurai, J. Zhou and S. Salous, "Low probability of intercept-based optimal power allocation scheme for an integrated multistatic radar and communication system," IEEE Systems Journal, vol. 14, no. 1, pp. 983-994, March 2020.

[43] Y. Zhou, H. Zhou, F. Zhou, Y. Wu and V. C. M. Leung, "Resource allocation for a wireless powered integrated radar and communication system," IEEE Wireless Communications Letters, vol. 8, no. 1, pp. 253256, Feb. 2019.

[44] B. Xiao, Y. Liu, K. Huo, J. Zhao and Z. Zhang, "OFDM integration waveform design for radar and communication application," in International Conference on Radar Systems (Radar 2017), Belfast, 2017.

[45] M. Biedka, Y. E. Wang, Q. M. Xu and Y. Li, "Full-duplex RF front ends: From antennas and circulators to leakage cancellation," IEEE Microwave Magazine, vol. 20, no. 2, pp. 44-55, Feb. 2019.

[46] S. A. Hassani, A. Guevara, K. Parashar, A. Bourdoux, B. van Liempd and S. Pollin, "An in-band full-duplex transceiver for simultaneous communication and environmental sensing," in Proc. Asilomar Conf. Signals Syst. Comput. (ASILOMAR), pp. 1389-1394, Oct. 2018.

[47] F. Shearer, "Power management in mobile devices," Communications Engineering Series, Newnes-Elsevier, 2008.

[48] S. K. Singh, K. Agrawal, K. Singh, C. Li and W. Huang, "On UAV selection and position-based throughput maximization in multi-UAV relaying networks," IEEE Access, vol. 8, pp. 144039-144050, 2020.

[49] C. Baquero Barneto et al., "Full-duplex OFDM radar with LTE and 5G NR waveforms: Challenges, solutions, and measurements," IEEE Transactions on Microwave Theory and Techniques, vol. 67, no. 10, pp. 4042-4054, Oct. 2019.

[50] Y. Du and P. Wei, "An explicit solution for target localization in noncoherent distributed MIMO radar systems," IEEE Signal Processing Letters, vol. 21, no. 9, pp. 1093-1097, Sept. 2014.

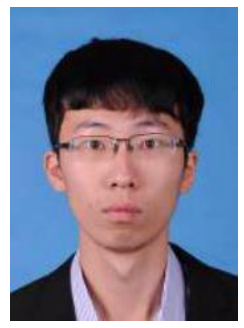

Xinyi Wang received the B.S. degree in electronic engineering from Beijing Institute of Technology (BIT), Beijing, China, in 2017. He is currently working toward the Master degree with the Research Institute of Communication Technology, BIT. His research interests include channel coding and modulation, mobile communication, joint communication and sensing.

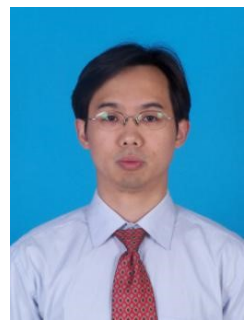

Zesong Fei (M07CSM16) received the Ph.D. degree in electronic engineering from the Beijing Institute of Technology (BIT), in 2004. Since September 2004, he has been with the School of Information and Electronics, Beijing Institute of Technology, Beijing, China, where he is currently a Full Professor. He has authored or co-authored more than 120 journal and conference articles. His current research interests include wireless communications, channel coding, multiple access, physical layer security, joint radar and communications, and MIMO systems.

Prof. Fei serves as an Associate Editor for the IEEE Access.

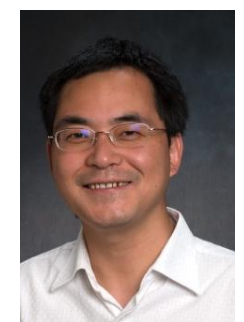

J. Andrew Zhang (M'04-SM'11) received the B.Sc. degree from Xi' an JiaoTong University, China, in 1996, the M.Sc. degree from Nanjing University of Posts and Telecommunications, China, in 1999, and the Ph.D. degree from the Australian National University, in 2004.

Currently, Dr. Zhang is an associate Professor in the School of Electrical and Data Engineering, University of Technology Sydney, Australia. He was a researcher with Data61, CSIRO, Australia from 2010 to 2016, the Networked Systems, NICTA, Australia from 2004 to 2010, and ZTE Corp, Nanjing, China from 1999 to 2001. Dr. Zhang's research interests are in the area of signal processing for wireless communications and sensing, and autonomous vehicular networks. He has published more than 190 papers in leading international Journals and conference proceedings, and has won 5 best paper awards for his work. $\mathrm{He}$ is a recipient of CSIRO Chairman's Medal and the Australian Engineering Innovation Award in 2012 for exceptional research achievements in multigigabit wireless communications.

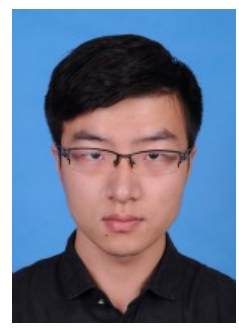

Jingxuan Huang received the B.S. degree in electronics engineering from the Beijing Institute of Technology (BIT), Beijing, China, in 2016. He is currently pursuing the Ph.D. degree with the Research Institute of Communication Technology, BIT. His research interests include channel coding and modulation, vehicular communications, and joint communication and sensing technology.

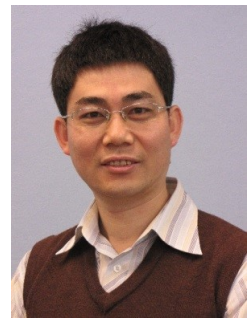

Jinhong Yuan (M'02-SM'11-F'16) received the B.E. and Ph.D. degrees in electronics engineering from the Beijing Institute of Technology, Beijing, China, in 1991 and 1997, respectively. From 1997 to 1999, he was a Research Fellow with the School of Electrical Engineering, University of Sydney, Sydney, Australia. In 2000, he joined the School of Electrical Engineering and Telecommunications, University of New South Wales, Sydney, Australia, where he is currently a Professor and Head of Telecommunication Group with the School. He has published two books, five book chapters, over 300 papers in telecommunications journals and conference proceedings, and 50 industrial reports. $\mathrm{He}$ is a co-inventor of one patent on MIMO systems and two patents on lowdensity-parity-check codes. He has co-authored four Best Paper Awards and one Best Poster Award, including the Best Paper Award from the IEEE International Conference on Communications, Kansas City, USA, in 2018 , the Best Paper Award from IEEE Wireless Communications and Networking Conference, Cancun, Mexico, in 2011, and the Best Paper Award from the IEEE International Symposium on Wireless Communications Systems, Trondheim, Norway, in 2007. He is an IEEE Fellow and currently serving as an Associate Editor for the IEEE Transactions on Wireless Communications. He served as the IEEE NSW Chapter Chair of Joint Communications/Signal Processions/Ocean Engineering Chapter during 2011-2014 and served as an Associate Editor for the IEEE Transactions on Communications during 2012-2017. His current research interests include error control coding and information theory, communication theory, and wireless communications. 